\title{
Bone Loss in Diabetes: Use of Antidiabetic Thiazolidinediones and Secondary Osteoporosis
}

\author{
Beata Lecka-Czernik
}

Published online: 1 September 2010

(C) The Author(s) 2010. This article is published with open access at Springerlink.com

\begin{abstract}
Clinical evidence indicates that bone status is affected in patients with type 2 diabetes mellitus (T2DM). Regardless of normal or even high bone mineral density, T2DM patients have increased risk of fractures. One class of antidiabetic drugs, thiazolidinediones (TZDs), causes bone loss and further increases facture risk, placing TZDs in the category of drugs causing secondary osteoporosis. Risk factors for development of TZD-induced secondary osteoporosis are gender (women), age (elderly), and duration of treatment. TZDs exert their antidiabetic effects by activating peroxisome proliferator-activated receptor- $\gamma$ (PPAR- $\gamma$ ) nuclear receptor, which controls glucose and fatty acid metabolism. In bone, PPAR- $\gamma$ controls differentiation of cells of mesenchymal and hematopoietic lineages. PPAR- $\gamma$ activation with TZDs leads to unbalanced bone remodeling: bone resorption increases and bone formation decreases. Laboratory research evidence points toward a possible separation of unwanted effects of PPAR- $\gamma$ on bone from its beneficial antidiabetic effects by using selective PPAR- $\gamma$ modulators. This review also discusses potential pharmacologic means to protect bone from detrimental effects of clinically used TZDs (pioglitazone and rosiglitazone) by using combinational therapy with approved antiosteoporotic drugs, or by using lower doses of TZDs in combination with other antidiabetic therapy. We also suggest a possible orthopedic complication, not yet supported by clinical studies, of delayed fracture healing in T2DM patients on TZD therapy.
\end{abstract}

B. Lecka-Czernik $(\bowtie)$

Departments of Orthopaedic Surgery and Physiology

and Pharmacology, Center for Diabetes and Endocrine Research,

University of Toledo Medical Center,

3000 Arlington Avenue,

Toledo, OH 43614, USA

e-mail: Beata.LeckaCzernik@utoledo.edu
Keywords Diabetes · Thiazolidinediones · Bone ·

Osteoporosis · Fracture

\section{Introduction}

Diabetes affects 180 million individuals worldwide with prevalence in all ages including very young children. The most common form is insulin-independent, or type 2 diabetes mellitus (T2DM). T2DM is characterized by insulin and glucose intolerance, and it is associated with development of hyperglycemia and hyperinsulinemia. Available therapies include insulin, insulin secretagogues (sulfonylureas), and glucose-lowering drugs (thiazolidinediones [TZDs], biguanide, and incretins) [1]. Out of these, only TZDs have insulin-sensitizing properties and are the most efficient for management of poorly controlled diabetes [1]. TZDs target peroxisome proliferator-activated receptor$\gamma($ PPAR $-\gamma)$ protein, a key regulator of energy metabolism in fat tissue. In response to the nutrients availability, such as glucose and fatty acids, PPAR- $\gamma$ controls energy storage and its dissipation by regulating lipogenesis and lipolysis [2]. These activities of PPAR- $\gamma$ include production of proteins involved in carbohydrate and fatty acids metabolism, as well as production of fat-specific cytokines, adipokines, which regulate insulin sensitivity in peripheral tissues. In bone, PPAR $\gamma$ controls bone cell development and the cytokine milieu of the bone marrow environment [3]. It regulates lineage commitment toward osteoblasts and adipocytes from common mesenchymal stem cells (MSCs), and bone resorbing osteoclasts from the pool of hematopoietic progenitors. In addition, PPAR- $\gamma$ controls production of a variety of cytokines, which support hematopoiesis, including receptor activator of nuclear factor- $\mathrm{KB}$ ligand (RANKL), which supports osteoclastogenesis. 


\section{Skeletal Status and Fracture Risk in T2DM}

The status of bone mineral density (BMD) and the risk of bone fractures in T2DM have been assessed in a number of studies. In general, individuals with T2DM have normal or increased BMD compared with nondiabetic patients; however, they have a higher incidence of bone fractures [4•]. Systematic analysis of 16 different well-controlled studies conducted in the United States and in Europe showed that T2DM was associated with a twofold increase in risk of hip fractures in men (relative risk [RR], 2.8) and women (RR, 2.1) [5]. Studies performed on a Japanese population indicated that T2DM patients, both women (odds ratio $[\mathrm{OR}], 1.83 ; P<0.01)$ and men (OR, 4.73; $P<0.001$ ), have increased rate of vertebral fractures [6]. Increased fracture risk is additionally elevated by diabetic complications including macrovascular complications, diabetic eye and kidney diseases, and neuropathy [7], which may lead to increased risk of trauma due to more frequent incidence of falls (RR, 1.64) [8]. In addition, factors such as duration of diabetic disease, aging, prior fracture, and corticosteroid use contribute to the greater fracture risk [9].

A lack of association between BMD and fracture risk suggests that diabetic bone has altered biomechanical quality. Human histomorphometric studies indicate that bone turnover in older T2DM patients is compromised, which may result in higher BMD but decreased bone quality [10]. Recent animal studies showed that high levels of insulin lead to high bone mass by decreasing both osteoclast number and bone resorption, and osteoblast number and bone formation [11]. Moreover, highly reactive glucose metabolites (advance glycation end products [AGEs]), of which circulating levels are increased in diabetes, are implicated in forming additional cross-links between collagen fibers in bone [12]. This process affects bone biomechanical properties by increasing its stiffness and fragility [13]. In support of this, recent studies showed a positive association between levels of circulating AGE pentosidine and increased incidence of fractures in diabetic patients $[12,14 \bullet]$.

\section{TZDs for Treatment of Type 2 Diabetes: Beneficial and Adverse Effects}

Two US Food and Drug Administration (FDA)-approved TZDs, rosiglitazone (Avandia; GlaxoSmithKline, Research Triangle Park, NC) and pioglitazone (Actos; Takeda Pharmaceuticals, Deerfield, IL), have been in clinical use since 1999. These drugs are used as glucose-lowering agents with a beneficial effect on insulin sensitivity. In addition to improving glycemic control in T2DM patients, they improve lipid metabolism with pioglitazone having a more favorable impact on serum lipids, lipoproteins, and apolipoproteins than rosiglitazone [15].

A number of studies showed superior efficacy of TZDs over other available antidiabetic therapies in the control of diabetic hyperglycemia [16]. However, their prolonged use is associated with several adverse effects. Strong clinical evidence points to the connection between rosiglitazone use and a significant increase in risk of myocardial infarction and death from cardiovascular causes [17]. This association resulted in a recent review of rosiglitazone safety by the FDA [18]. Interestingly, pioglitazone use is associated with a significantly lower risk of death and lower number of myocardial infarction and stroke incidence [19], indicating that cardiovascular effects of TZDs are not a drug class effect, but rather specifically associated with the TZD type. Both TZDs exhibit drug class properties of fluid retention and weight gain [20].

\section{TZD's Effect on Bone: Clinical Research Evidence}

There is accumulating evidence that both rosiglitazone and pioglitazone cause bone loss and increase fracture risk in humans, specifically in women [4•]. Observational studies using data from the Health, Aging, and Body Composition cohort reported that older postmenopausal TZD users experience bone loss at the rate of $-0.61 \%$ annually compared with non-TZD users [21]. In Japanese patients with $\mathrm{T} 2 \mathrm{DM}$, treatment for 1 year with pioglitazone decreased serum osteocalcin, femoral and radial BMD, but not BMD in lumbar vertebra [22]. Bone loss was also observed with a short treatment with TZDs. A randomized, placebo-controlled study of the effect of pioglitazone on bone in polycystic ovary syndrome patients in Denmark demonstrated that 16 weeks of treatment resulted in a significant decline in BMD of the lumbar spine $(-1.1 \%)$ and femoral neck $(-1.4 \%)$ [23]. Similarly, a randomized controlled trial of rosiglitazone effects on bone of postmenopausal nondiabetic women in New Zealand showed that 14 weeks of rosiglitazone administration resulted in a decrease in hip BMD by $-1.9 \%$ compared with the BMD at the beginning of treatment [24]. Changes in BMD were accompanied by a decrease in serum markers of bone formation, such as alkaline phosphatase and aminoterminal propeptide of type I collagen (P1NP). Bone resorption markers lacked change, leading to the conclusion that shortterm therapy with rosiglitazone exerts detrimental skeletal effects by inhibiting bone formation [24]. In contrast, 16 weeks of treatment with rosiglitazone of T2DM women with a prior diagnosis of cardiovascular disease resulted in significantly increased levels of the circulating bone resorption marker $\mathrm{C}$-terminal collagen crosslinked peptide 
(CTX), whereas levels of P1NP were not changed [25]. The same studies showed that rosiglitazone did not affect markers of bone turnover in men [25]. The contrasting results of studies by Grey et al. [24] and Gruntmanis et al. [25] suggest that bone response to TZDs may be determined by hormonal and metabolic status.

The causal connection between TZD therapy and increased fracture risk was determined in a number of studies, the majority of which were retrospective. The analysis of these studies allows for defining of risk factors to increased fractures in TZD users such as gender, age, pre-existing conditions, and duration of treatment. The first demonstration of increased fracture risk was noticed during an analysis of results from ADOPT (A Diabetes Outcome Progression Trial), which was designed to compare an efficacy of different antidiabetic therapy on maintenance of normal glucose levels in prediabetic individuals [16]. Posttrial data from the 1,840 women and 2,511 men randomly assigned in ADOPT to rosiglitazone, metformin, or glyburide for a median of 4.0 years were examined with respect to time to first fracture, rates of occurrence, and sites of fractures $\left[16,26^{\circ}\right]$. Fracture rate in men did not differ between treatment groups and did not demonstrate significant difference in an overall risk. The cumulative incidence of fractures in women was $15.1 \%(11.2-19.1)$ with rosiglitazone, $7.3 \%(4.4-10.1)$ with metformin, and $7.7 \%$ (3.7-11.7) with glyburide, representing hazard ratios of 1.81 and 2.13 for rosiglitazone compared with metformin and glyburide, respectively. Fractures were seen predominantly in the lower and upper limbs, and vertebral fractures were not assessed in this study. Increased fracture rate in women correlated with increased levels in serum of bone resorption marker (CTX) but not bone formation marker (P1NP) [27]. There was no correlation between rosiglitazone use and estrogen status since both pre- and postmenopausal women demonstrated increase in fractures [16, 26•]. These observations were subsequently corroborated by a number of other studies. A meta-analyses of data from 10 different randomized controlled trials involving 13,715 participants and two observational studies involving 31,679 participants confirmed that long-term TZD use doubles the risk of fractures exclusively in women but not in men with T2DM [28•]. Recently, the cross-sectional study based on use of medical and pharmacy claims for TZD prescriptions and involving the southeastern region of the United States showed that TZD use, regardless of type, doubled the incidence of distal upper and lower limb fracture, and the proportion was significantly higher in women and increased $2 \%$ for every year increase in age [29]. Similarly, retrospective studies involving 19,070 individuals of both genders in southeast Michigan showed that women more than 65 years of age appeared to be at greatest risk for fractures [30].
In contrast, retrospective studies on 84,339 diabetic patients in British Columbia (Canada) concluded that both women and men receiving TZDs have increased fracture risk. The risk further increased with duration of treatment, and pioglitazone was more strongly associated with fractures than rosiglitazone, especially in men [31]. Observational studies based on the UK General Practice Research Database (GPRD), which included a large population of older individuals, showed that TZD therapy and its duration are associated with significant increase in nonvertebral fractures independent of patient sex and age. The adjusted OR of fracture occurrence for hip/femur was 4.54 , for humerus was 2.12, and for wrist/forearm was 2.90 [32]. Studies conducted on a cohort of Medicare beneficiaries with diabetes over 65 years of age and older showed that compared with sulfonylureas and metformin monotherapies, TZD monotherapy is associated with increased risk of peripheral fractures regardless of sex and type of TZD [33].

Another self-controlled case-series study on the GPRD population suggested that prior fracture(s) increases the risk of the next fracture occurrence. These studies compared rates of fractures within the person with prior fracture during TZD exposed and unexposed periods and showed that exposure to TZD, either rosiglitazone or pioglitazone, increased fracture rate by $43 \%$ similarly in men and women and the duration of exposure increased this risk even further [34]. Fractures occurred in a range of sites including hip, spine, arm, foot, wrist, and hand.

Taken together, results of available studies indicate the following: 1) TZD effect on bone is a drug class effect; 2) women and elderly are at increased risk of bone loss and increased risk of fractures; however, some studies point to the equal risk in both genders; 3 ) the risk is increased in individuals who have a history of prior TZD-unrelated fractures; and 4) duration of treatment correlates positively with increased fracture risk.

On a positive note, TZD's negative effect on bone can be beneficial in treatment of conditions of increased bone formation. Treatment with rosiglitazone of an acromegalic patient with T2DM significantly decreased serum levels of growth hormone and insulin-like growth factor-1 (IGF-1), and improved acromegalic features [35]. This clinical observation points to the possibility of using TZDs in patients with pituitary tumors associated with increased growth hormone production. Another case report suggests that TZDs may be used to slow down progress of fibrodysplasia ossificans progressiva (FOP). FOP is a genetic disease characterized by heterotopic bone formation due to upregulation of bone morphogenetic protein (BMP) signaling in response to inflammatory input [36]. It has been reported that rosiglitazone not only prevented new flares of heterotopic bone formation, but also improved general mobility of the FOP patient [36]. TZD's negative 
effects on bone may benefit treatment of conditions of increased bone formation.

\section{TZD's Effect on Bone: Laboratory Research Evidence}

TZDs bind to and activate the PPAR- $\gamma$ transcription factor, which is a key regulator of bone cell development and their activity in the adult skeleton [3]. In animal models, TZDs induce bone loss by affecting the bone remodeling process; TZDs suppress new bone formation by osteoblasts and increase bone resorption by osteoclasts. Age and sex steroid status determine which mechanism contributes predominantly to the observed bone loss. An administration of rosiglitazone to mice for a period of 8 weeks resulted in significant decreases in BMD, bone volume, and changes in bone microarchitecture [37, 38]. Observed bone loss was associated with changes in the structure and function of bone marrow, which included decreased number of osteoblasts, increased number of adipocytes, and increased support for osteoclastogenesis. The degree of bone loss in response to rosiglitazone correlated with the animal age and the level of PPAR- $\gamma$ expression in bone [38]. In younger animals with less PPAR- $\gamma$, trabecular bone loss in the proximal tibia was associated with a decreased number of osteoblasts and a reduced bone formation rate. In older animals with higher levels of PPAR- $\gamma$, bone loss correlated with an increased number of osteoclasts and increased bone resorption, whereas the quantity of osteoblasts and the bone formation rate remained unchanged [38]. Similarly, estrogen deficiency influences TZD-induced bone loss in murine models. Studies of rosiglitazone effects in estrogendeficient rats and mice showed that bone loss occurred mainly due to increased bone resorption [39, 40], pointing to the functional cross-talk between PPAR- $\gamma$ and estrogen receptor. Such cross-talk has been recently described in osteoblasts as a form of competition between PPAR- $\gamma$ and estrogen receptor for a common coactivator, steroid receptor coactivator-2 (Src-2) [41]. In conclusion, animal studies suggest that aging and estrogen deficiency confound TZD-induced bone loss and determine its mechanism.

Two mechanisms may contribute to increased bone resorption: an increased production by mesenchymal cells of osteoclastogenesis supporting cytokine RANKL and activation of PPAR- $\gamma$ in cells of hematopoietic lineage $[38,42]$. As showed recently, PPAR- $\gamma$ promotes osteoclast recruitment and differentiation from hematopoietic progenitors by controlling an expression of c-fos protein, an important determinant of osteoclast lineage commitment and development $[42,43]$. Mice deficient in PPAR- $\gamma$ expression in cells of hematopoietic lineage develop osteopetrosis and are less sensitive to the TZD-induced bone loss than control mice [42].

TZD's negative effects on bone formation raise a concern regarding their effects on bone healing. Studies from our laboratory have shown that TZDs interfere with new bone formation at the bone healing site. In a distraction osteogenesis model of intramembraneous bone formation, we have shown that rosiglitazone significantly decreases endosteal bone formation. This effect is associated with a decreased number of bone-forming osteoblasts, decreased new vessel formation, and accumulation of large quantities of fat at the healing site (Lecka-Czernik, Unpublished data). The negative effect of TZDs on fracture healing should be addressed in clinical studies, and if confirmed, a discontinuation of TZD therapy during the period of fracture healing should be considered.

Studies on molecular and cellular mechanisms activated by TZDs suggest that in marrow cells of mesenchymal lineage TZDs suppress bone anabolic signaling by decreasing activity of Wnt, transforming growth factor- $\beta$ (TGF- $\beta$ )/ BMP, and IGF-1 pathways, while inducing production of RANKL, a cytokine supporting osteoclast development $[38,44 \cdot]$. The effects on activities of TGF- $\beta$ /BMP and IGF1 pathways may explain in part the beneficial effects of TZDs on treatment of FOP and acromegaly, as mentioned above [35]. The decrease in activity of osteoblast-specific signaling pathways precedes the negative effects of TZDs on the expression of genes essential for osteoblast development, such as Runx2, Dlx5, and Osterix. Moreover, TZDs affect the expression of genes involved in the maintenance of MSC-like phenotype, so-called "stemness" genes such as LIF and c-kit, while inducing the expression of adipocyte-specific genes [45 ${ }^{\bullet}$. As a result, MSCs lose their potential to differentiate toward osteoblasts. Instead, they acquire an adipocyte phenotype. These features resemble changes that occur during MSC aging, and which result in a decreased number of osteoblasts and bone formation, and accumulation of fat in bone marrow [46, 47]. Moreover, the number of osteoclasts and production of RANKL increases with aging, and results in increased bone resorption [48]. Because TZD-induced changes in bone marrow structure/function are strikingly similar to changes that occur during aging, some speculate that TZDs accelerate the aging of bone $[38,46]$.

\section{Selective PPAR- $\gamma$ Modulators: Ligand-Dependent Separation of Proadipocytic and Antiosteoblastic Activities of PPAR- $\gamma$}

The PPAR $\gamma$ ligand-binding domain contains a large binding pocket capable of encompassing a variety of 
ligands. This provides a wide array of potential contact points that can result in various PPAR- $\gamma$ conformations and differential recruitment of coactivators, which determine specificity of this nuclear receptor [2]. Although TZDs possess a beneficial antidiabetic profile, their adverse effects prompt pharmaceutical efforts to develop selective PPAR- $\gamma$ modulators that will retain high potency to treat diabetic disease with minimal adverse effects [49].

The molecular studies provide evidence for distinct regulatory pathways that regulate proadipocytic and antiosteoblastic activities of PPAR- $\gamma$. These studies demonstrated that PPAR- $\gamma$ proadipocytic activity is transcriptional in nature and involves binding to PPAR response elements (PPRE) in gene regulatory regions, whereas its antiosteoblastic activity is PPRE-independent [44•]. PPAR- $\gamma$ anti-inflammatory and antiatherogenic activities are also regulated in a PPREindependent manner [50].

With respect to bone, it has been demonstrated that PPAR- $\gamma$ proadipocytic and antiosteoblastic activities can be separated by using ligands of different chemical structures [51]. In an in vitro model of marrow mesenchymal cell differentiation under control of PPAR- $\gamma$, ligands consisting of several structurally related oxidized derivatives of linoleic acid were able to activate all three combinations of PPAR- $\gamma$ activity: proadipocytic, antiosteoblastic, or both. In addition, we have shown that the TZD netoglitazone, which has antihyperglycemic properties comparable with rosiglitazone, does not suppress osteoblastogenesis. Mice receiving netoglitazone at the dose that decreased glucose levels similarly to rosiglitazone did not lose bone [52].

\section{Can We Prevent Secondary Osteoporosis in TZD Users?}

Clinical evidence indicating an increased risk of bone loss and fractures in TZD users requires more research addressing an improvement to bone safety. Advances in development of new pharmacologic means to treat diabetes are broadening the arsenal of drugs that target different but complementary mechanisms of glucose metabolism. Combination therapies that focus on different mechanisms promise better pharmacologic outcome with a possibility to reduce doses of each particular drug. A combination of TZDs with metformin reduces the dose of TZDs by half and was shown to control hyperglycemia, hemoglobin $\mathrm{A}_{1 \mathrm{c}}$ $\left(\mathrm{HbA}_{1 \mathrm{c}}\right)$ levels, and prevent progression to diabetes of prediabetic individuals [53]. Both rosiglitazone and pioglitazone are available in such combinations known as Avandamet (GlaxoSmithKline) and ACTOplus met (Takeda Pharmaceuticals), respectively. Another possibility is a combination of TZDs with incretin-based therapy. Although
TZDs have a negative effect on bone, incretins seem to have a positive effect by stimulating anabolic activities of osteoblasts and suppressing catabolic activities of osteoclasts [54]. From the diabetic benefit point of view, it has been demonstrated that TZDs in combination with glucagon-like peptide-1 analogues, or with dipeptidyl peptidase-4 serine protease inhibitors, allows for better control of hyperglycemia and $\mathrm{HbA}_{1 \mathrm{c}}$ levels [55]. However, the safety for bone of such combined therapies has not been investigated as yet.

Whereas combined therapy with other antidiabetic drugs may minimize a negative effect on bone, the therapy in which TZDs are administered in combination with antiosteoporotic drugs (eg, bisphosphonates) may protect bone. Administration of alendronate with pioglitazone to aging mice completely protected bone from its loss compared with the group of animals receiving pioglitazone alone (Lecka-Czernik, Unpublished data). In view of recent unfavorable reviews of rosiglitazone safety, it is possible that a competitor pioglitazone will remain on the market as the only available TZD. Taking into account its continued use because of its beneficial effect for control of diabetes, more research has to be done to improve bone safety during pioglitazone therapy.

\section{Conclusions}

The available evidence indicates that antidiabetic TZD therapy is associated with bone loss and increased fracture risk. The mechanism of TZD-induced bone loss includes unbalanced bone remodeling processes resulting from decreased bone formation and increased bone resorption. Human and animal studies suggest that aging and estrogen deficiency confound TZD-induced bone loss and determine its mechanism. Improvement of bone safety of TZD-based therapy may be achieved by using combination therapy with other antidiabetic therapies, or preventing bone loss by using available antiosteoporotic drugs, or development of a new class of selective TZDs, which may have sparing or even beneficial effects on skeleton. A new provocative question is the effect of TZDs on fracture healing in diabetic patients. This question requires clinical studies to assess a risk of orthopedic complications in TZD users, and may caution against continuation of TZD therapy during a period of fracture healing.

Acknowledgments This review was supported by funds from NIH/ NIA AG 028935 and American Diabetes Association's Amaranth Diabetes Fund 1-09-RA-95.

Disclosure No potential conflict of interest relevant to this article was reported. 
Open Access This article is distributed under the terms of the Creative Commons Attribution Noncommercial License which permits any noncommercial use, distribution, and reproduction in any medium, provided the original author(s) and source are credited.

\section{References}

Papers of particular interest, published recently, have been highlighted as:

- Of importance

1. Cheng AY, Fantus IG: Oral antihyperglycemic therapy for type 2 diabetes mellitus. CMAJ 2005, 172:213-226.

2. Tontonoz P, Spiegelman BM: Fat and beyond: the diverse biology of PPARgamma. Annu Rev Biochem 2008, 77:289-312.

3. Lecka-Czernik B: PPARs in bone: the role in bone cell differentiation and regulation of energy metabolism. Curr Osteoporos Rep 2010, 8:84-90.

4. - Lecka-Czernik B: Bone as a target of type 2 diabetes treatment. Curr Opin Investig Drugs 2009, 10:1085-1090. This article reviews the effect of T2DM and different antidiabetic therapies on the human skeleton.

5. Janghorbani M, Van Dam RM, Willett WC, Hu FB: Systematic review of type 1 and type 2 diabetes mellitus and risk of fracture. Am J Epidemiol 2007, 166:495-505.

6. Yamamoto M, Yamaguchi T, Yamauchi M, et al.: Diabetic patients have an increased risk of vertebral fractures independent of BMD or diabetic complications. J Bone Miner Res 2009, 24: 702-709.

7. Vestergaard P, Rejnmark L, Mosekilde L: Diabetes and its complications and their relationship with risk of fractures in type 1 and 2 diabetes. Calcif Tissue Int 2009, 84:45-55.

8. Schwartz AV, Hillier TA, Sellmeyer DE, et al.: Older women with diabetes have a higher risk of falls: a prospective study. Diabetes Care 2002, 25:1749-1754.

9. Melton LJ, Leibson CL, Achenbach SJ, et al.: Fracture risk in type 2 diabetes: update of a population-based study. J Bone Miner Res 2008, 23:1334-1342.

10. Krakauer JC, McKenna MJ, Buderer NF, et al.: Bone loss and bone turnover in diabetes. Diabetes 1995, 44:775-782.

11. Huang S, Kaw M, Harris MT, et al.: Decreased osteoclastogenesis and high bone mass in mice with impaired insulin clearance due to liver-specific inactivation to CEACAM1. Bone 2010, 46:1138-1145.

12. Yamamoto $M$, Yamaguchi $T$, Yamauchi $M$, et al.: Serum pentosidine levels are positively associated with the presence of vertebral fractures in postmenopausal women with type 2 diabetes. J Clin Endocrinol Metab 2008, 93:1013-1019.

13. Vashishth D: Collagen glycation and its role in fracture properties of bone. J Musculoskelet Neuronal Interact 2005, 5:316.

14. - Schwartz AV, Garnero P, Hillier TA, et al.: Pentosidine and increased fracture risk in older adults with type 2 diabetes. J Clin Endocrinol Metab 2009, 94:2380-2386. This study shows association between levels of circulating AGEs and increased fracture risk in T2DM patients.

15. Deeg MA, Tan MH: Pioglitazone versus rosiglitazone: effects on lipids, lipoproteins, and apolipoproteins in head-to-head randomized clinical studies. PPAR Res 2008, 2008:520465.

16. Kahn SE, Haffner SM, Heise MA, et al.: Glycemic durability of rosiglitazone, metformin, or glyburide monotherapy. N Engl J Med 2006, 355:2427-2443.

17. Nissen SE, Wolski K: Effect of rosiglitazone on the risk of myocardial infarction and death from cardiovascular causes. $\mathrm{N}$ Engl J Med 2007, 356:2457-2471.
18. Wadman M: Avandia outcome may signal change in epidemiologists' sway. Nat Med 2010, 16:614.

19. Lincoff AM, Wolski K, Nicholls SJ, Nissen SE: Pioglitazone and risk of cardiovascular events in patients with type 2 diabetes mellitus: a meta-analysis of randomized trials. JAMA 2007, 298:1180-1188.

20. Yki-Jarvinen H: Thiazolidinediones. N Engl J Med 2004, 351:1106-1118.

21. Schwartz AV, Sellmeyer DE, Vittinghoff E, et al.: Thiazolidinedione (TZD) use and bone loss in older diabetic adults. J Clin Endocrinol Metab 2006, 91:3349-3354.

22. Kanazawa I, Yamaguchi T, Yano S, et al.: Baseline atherosclerosis parameter could assess the risk of bone loss during pioglitazone treatment in type 2 diabetes mellitus. Osteoporos Int 2010 Feb 4 [Epub ahead of print].

23. Glintborg D, Andersen M, Hagen C, et al.: Association of pioglitazone treatment with decreased bone mineral density in obese premenopausal patients with polycystic ovary syndrome: a randomized, placebo-controlled trial. J Clin Endocrinol Metab 2008, 93:1696-1701.

24. Grey A, Bolland M, Gamble G, et al.: The peroxisomeproliferator-activated receptor-gamma agonist rosiglitazone decreases bone formation and bone mineral density in healthy postmenopausal women: a randomized, controlled trial. J Clin Endocrinol Metab 2007, 92:1305-1310.

25. Gruntmanis U, Fordan S, Ghayee HK, et al.: The peroxisome proliferator-activated receptor-gamma agonist rosiglitazone increases bone resorption in women with type 2 diabetes: a randomized, controlled trial. Calcif Tissue Int 2010, 86:343-349.

26. • Kahn SE, Zinman B, Lachin JM, et al.: Rosiglitazone-associated fractures in type 2 diabetes: an Analysis from A Diabetes Outcome Progression Trial (ADOPT). Diabetes Care 2008, 31:845-851. This article provides a retrospective analysis of fracture risk and type of fractures among the ADOPT participants receiving rosiglitazone.

27. Zinman B, Haffner SM, Herman WH, et al.: Effect of rosiglitazone, metformin, and glyburide on bone biomarkers in patients with type 2 diabetes. J Clin Endocrinol Metab 2010, 95:134-142.

28. - Loke YK, Singh S, Furberg CD: Long-term use of thiazolidinediones and fractures in type 2 diabetes: a meta-analysis. CMAJ 2009, 180:32-39. This article provides a comprehensive review of available clinical trials in respect to the effect of TZDs on risk of fractures.

29. Jones SG, Momin SR, Good MW, et al.: Distal upper and lower limb fractures associated with thiazolidinedione use. Am J Manag Care 2009, 15:491-496.

30. Habib ZA, Havstad SL, Wells K, et al.: Thiazolidinedione use and the longitudinal risk of fractures in patients with type 2 diabetes mellitus. J Clin Endocrinol Metab 2010, 95:592-600.

31. Dormuth CR, Carney G, Carleton B, et al.: Thiazolidinediones and fractures in men and women. Arch Intern Med 2009, 169:1395-1402.

32. Meier C, Kraenzlin ME, Bodmer M, et al.: Use of thiazolidinediones and fracture risk. Arch Intern Med 2008, 168:820-825.

33. Solomon DH, Cadarette SM, Choudhry NK, et al.: A cohort study of thiazolidinediones and fractures in older adults with diabetes. J Clin Endocrinol Metab 2009, 94:2792-2798.

34. Douglas IJ, Evans SJ, Pocock S, Smeeth L: The risk of fractures associated with thiazolidinediones: a self-controlled case-series study. PLoS Med 2009, 6:e1000154.

35. Gradiser M, Matovinovic M, Vrkljan M: Decrease in growth hormone and insulin-like growth factor (IGF)-1 release and amelioration of acromegaly features after rosiglitazone treatment of type 2 diabetes mellitus a patient with acromegaly. Croat Med J 2007, 48:87-91.

36. Gatti D, Viapiana O, Rossini M, Silvano A: Rosiglitazone therapy is associated with major clinical improvements in a patient with 
fibrodysplasia ossificans progressive. J Bone Miner Res 2010, 25:1460-1462.

37. Rzonca SO, Suva LJ, Gaddy D, et al.: Bone is a target for the antidiabetic compound rosiglitazone. Endocrinology 2004, 145:401-406.

38. Lazarenko OP, Rzonca SO, Hogue WR, et al.: Rosiglitazone induces decreases in bone mass and strength that are reminiscent of aged bone. Endocrinology 2007, 148:2669-2680.

39. Sottile V, Seuwen K, Kneissel M: Enhanced marrow adipogenesis and bone resorption in estrogen-deprived rats treated with the PPARgamma agonist BRL49653 (rosiglitazone). Calcif Tissue Int 2004, 75:329-337.

40. Huang S, Syed F, Suva LJ, Lecka-Czernik B: Estrogen deficiency augments TZD-induced bone loss and fat accumulation in bone in vivo [abstract]. J Bone Miner Res 2009, 24(Suppl 1). Available at http://www.asbmr.org/Meetings/AnnualMeeting/AbstractDetail. aspx?aid=2e0a4c8c-af53-41a8-8155-8818183333bc. Accessed August 2010.

41. Modder UI, Monroe DG, Fraser DG, et al.: Skeletal consequences of deletion of steroid receptor coactivator-2/transcription intermediary factor-2. J Biol Chem 2009, 284:18767-18777.

42. Wan Y, Chong LW, Evans RM: PPAR-gamma regulates osteoclastogenesis in mice. Nat Med 2007, 13:1496-1503.

43. Teitelbaum SL, Ross FP: Genetic regulation of osteoclast development and function. Nat Rev Genet 2003, 4:638-649.

44. - Shockley KR, Lazarenko OP, Czernik PJ, et al.: PPARgamma2 nuclear receptor controls multiple regulatory pathways of osteoblast differentiation from marrow mesenchymal stem cells. J Cell Biochem 2009, 106:232-246. This article provides an extensive analysis of the effect of rosiglitazone on gene expression in marrow MSCs.

45. - Shockley KR, Rosen CJ, Churchill GA, Lecka-Czernik B: PPARgamma2 regulates a molecular signature of marrow mesenchymal stem cells. PPAR Res 2007, 2007:81219. This article demonstrates that PPAR- $\gamma 2$ plays a major role in regulation of cell "stemness."
46. Moerman EJ, Teng K, Lipschitz DA, Lecka-Czernik B: Aging activates adipogenic and suppresses osteogenic programs in mesenchymal marrow stroma/stem cells: the role of PPAR-gamma2 transcription factor and TGF-beta/BMP signaling pathways. Aging Cell 2004, 3:379-389.

47. Rosen CJ, Bouxsein ML: Mechanism of disease: is osteoporosis the obesity of bone? Nat Clin Pract Rheumatol 2006, 2:35-43.

48. Cao JJ, Wronski TJ, Iwaniec U, et al.: Aging increases stromal/ osteoblastic cell-induced osteoclastogenesis and alters the osteoclast precursor pool in the mouse. J Bone Miner Res 2005, 20:1659-1668.

49. Higgins LS, Depaoli AM: Selective peroxisome proliferatoractivated receptor gamma (PPARgamma) modulation as a strategy for safer therapeutic PPARgamma activation. Am J Clin Nutr 2010, 91:267 S-272 S.

50. Glass CK, Saijo K: Nuclear receptor transrepression pathways that regulate inflammation in macrophages and $\mathrm{T}$ cells. Nat Rev Immunol 2010, 10:365-376.

51. Lecka-Czernik B, Moerman EJ, Grant DF, et al.: Divergent effects of selective peroxisome proliferator-activated receptor-gamma 2 ligands on adipocyte versus osteoblast differentiation. Endocrinology 2002, 143:2376-2384.

52. Lazarenko OP, Rzonca SO, Suva LJ, Lecka-Czernik B: Netoglitazone is a PPAR-gamma ligand with selective effects on bone and fat. Bone 2006, 38:74-85.

53. Stafford JM, Elasy T: Treatment update: thiazolidinediones in combination with metformin for the treatment of type 2 diabetes. Vasc Health Risk Manag 2007, 3:503-510.

54. Wong IP, Baldock PA, Herzog H: Gastrointestinal peptides and bone health. Curr Opin Endocrinol Diabetes Obes 2010, 17:44-50.

55. Zinman B, Gerich J, Buse JB, et al.: Efficacy and safety of the human glucagon-like peptide-1 analog liraglutide in combination with metformin and thiazolidinedione in patients with type 2 diabetes (LEAD-4 Met+TZD). Diabetes Care 2009, 32:1224-1230. 